\title{
A NOVEL IMAGE ACQUISITION AND PROCESSING PROCEDURE FOR FAST TUNNEL DSM PRODUCTION
}

\author{
R. Roncella, G. Umili, G. Forlani \\ DICATeA, University of Parma, 43124 Parma, Italy - (riccardo.roncella, gessica.umili, gianfranco.forlani)@unipr.it
}

Commission V, WG V/4

KEY WORDS: DEM/DTM, Restitution, Automation, Orthoimage, Close Range, Photogrammetry

\begin{abstract}
:
In mining operations the evaluation of the stability condition of the excavated front are critic to ensure a safe and correct planning of the subsequent activities. The procedure currently used to this aim has some shortcomings: safety for the geologist, completeness of data collection and objective documentation of the results. In the last decade it has been shown that the geostructural parameters necessary to the stability analysis can be derived from high resolution digital surface models (DSM) of rock faces. With the objective to overcome the limitation of the traditional survey and to minimize data capture times, so reducing delays on mining site operations, a photogrammetric system to generate high resolution DSM of tunnels has been realized. A fast, effective and complete data capture method has been developed and the orientation and restitution phases have been largely automated. The survey operations take no more than required to the traditional ones; no additional topographic measurements other than those available are required. To make the data processing fast and economic our Structure from Motion procedure has been slightly modified to adapt to the peculiar block geometry while, the DSM of the tunnel is created using automatic image correlation techniques. The geomechanical data are sampled on the DSM, by using the acquired images in a GUI and a segmentation procedure to select discontinuity planes. To allow an easier and faster identification of relevant features of the surface of the tunnel, using again an automatic procedure, an orthophoto of the tunnel is produced. A case study where a tunnel section of ca. $130 \mathrm{~m}$ has been surveyed is presented.
\end{abstract}

\section{INTRODUCTION}

\subsection{Geostructural data acquisition in tunnels}

The mining operations in an underground quarry run continuously all over the day and are organized in cycles each made by three phases: the perforation and the insertion of the explosive charges at the front, the detonation of the charges and the removal of the material. Since the drilling machine is guided by a laser system, points are marked along the walls of the tunnel and surveyed with a total station so that any subsequent operation can be referred to the tunnel reference system, monitoring the progress of the excavation and checking its conformity to plans.

Technical prescriptions foresee that a geological report is produced which describes the general geostructural characteristics found during the progress of excavations. Periodically, a geological survey is therefore executed to verify the stability conditions at the front. The ISRM (International Society for Rock Mechanics) recommendations prescribe the sampling of all the discontinuities traces that intersect a certain reference line (scan line) or that fall within a certain window: for every discontinuity track detected the orientation is assessed using a geological compass and the spacing with respect to traces of the same family is measured with a tape and reported in a sketch.

The gathered data are analysed generally using a statistical approach: by clustering all the spatial orientations of the discontinuities, their families are highlighted and the behaviour in case of collapse of the rock structure predicted. It is therefore essential that the number of discontinuities sampled is high enough to make the statistical analysis significant. Moreover, the geologist must evaluate the lithology of the rock mass, the presence of water and/or gas and carry out tests of resistance to compression.
Though routinely applied, this method has some drawbacks. Apart from the risk of falling stones in an area not yet consolidated, the measurement are restricted to the lower part of the tunnel, directly accessible by the geologist. The survey takes some time to carry out, which interrupts the excavation activities. Moreover, if the tunnel is intended for civil use (e.g. a road or a railway), the documentation gathered by the geologists cannot be verified later. In case of an accident due to rock fall during the construction or other damages occurring after the opening to traffic it's almost impossible to independently assess the actual initial geomorphological conditions and find out whether some design or construction error occurred: in other words it is not possible to verify the correspondence of the measured data with the previous state of the tunnel.

Improving the data collection in terms of safety, amount and distribution of measurements without increasing or perhaps even reducing the time needed would improve the efficiency of the quarry management; a better documentation of the surveys would also be desirable.

To improve the data acquisition technique, overcoming its current critical aspect, it was decided to develop an innovative system based on photogrammetry, where the geostructural characteristics are derived from measurement made on a high resolution DSM (Digital Surface Model).

\subsection{Non-contact measurement techniques}

So far the use of photogrammetric techniques in tunnels has been concentrated on the monitoring of the deformations and displacements in the period following the excavation, measuring periodically the three-dimensional displacements of targets applied to the vault and to the side walls (Nakai et al., 2003). The same operation are today mostly performed using a Terrestrial Laser Scanner (TLS) (Nuttens, T. et al., 2010; Fekete et al., 2009) comparing point clouds acquired at different 
epochs. Recently commercial close-range photogrammetric software designed for the generation of digital models of tunnels have been produced (Somervuori \& Lamberg, 2009).

As far as mapping the discontinuities traces is concerned, images has been used for some time (Tsoutrelis et al., 1990; Crosta, 1997); with image processing techniques traces are extracted and measured automatically in underground operations, where lighting conditions can be controlled (Hadjigeorgiou and Lemy, 2005). Discontinuity traces are particularly important in stability analysis of tunnels since the discontinuity surfaces are always very small and expertise is required in discriminating structural discontinuities due to the disturbance of blasting and excavation. Determination of discontinuity planes by segmentation of point clouds or analysis of a DSM have been proposed and used for some time in the geological survey of open pits and rock faces (Roncella and Forlani, 2005; Slob et al, 2005)

\section{SYSTEM DESCRIPTION}

The system developed is born with the aim of allowing a threedimensional reconstruction of the detail of the whole tunnel (walls and ceilings of excavation vaults and facade) requiring, however, short acquisition times to not interfere excessively with the excavation operations. The galleries are considered 8 to $12 \mathrm{~m}$ (mining tunnels) wide with approximately vertical piers with a height of 3 to 5 meters supporting a semi-circular vault: the total height of the tunnel is thus between 7 and 11 meters.

Using a TLS in such environment requires much longer data acquisition times compare to photogrammetry, if uniformity in object sampling resolution and minimization of occlusions should be achieved on the very irregular surface of the tunnel. Indeed, the only way to comply with such requirement would be setting TLS stations at very short distances; this takes time for setting the instrument, orienting the scans, as well as, at least for some instruments, for data acquisition: overall, no less than 1015 minutes per station, even to an experienced operator.

Photogrammetry is therefore considered a better approach to the problem: image acquisition is usually very fast and moving a digital camera is much easier than a heavy TLS; even if the number of the photo stations is high, the time required to shoot the images are minor if compared to the other analysis the geologists should perform; the system developed is able to significantly reduce the time of in situ survey, assigning to successive stages of data processing the restitution of the model.

In particular a prototype of a panoramic head mounted on a photographic or topographic tripod has been developed allowing easy movement of equipment (digital reflex camera) and allowing, at the same time, to meet the block geometry specifications granting the required accuracy and resolution of the DSM.

As far as image data analysis is concerned, to limit the intervention of human operators during the block orientation and DSM restitution, proprietary developed Structure from Motion (SfM) and Dense Matching (DM) algorithms have been adapted to make these phases completely automated. In this way the same product obtainable by TLS (a high resolution DSM of the gallery) is produced in shorter times and at low costs. These aspects, which are probably the most technically and scientifically significant contribution of the paper, will be treated in the next section.

\subsection{Image acquisition device}

The device developed for fast and effective image acquisition is made of a digital camera, a laser pointer and a photographic tripod with a panoramic head. The tripod is set to position the camera at an appropriate elevation from ground; the head allows to rotate the camera around a horizontal axis by $180^{\circ}$; a series of stops allows the rotation of the camera by predefined angular steps, designed to maintain the desired overlap between consecutive shots (see Figure 1.a).

The tripod is positioned in such a way that the camera projection center is approximately in the longitudinal plane of symmetry of the tunnel. The laser pointer, aligned to the axis of rotation of the device, help to set the tripod in the correct position, by moving the head untill the laser spot is on a painted cross on the excavation front that marks the tunnel axis. The imaging geometry foresees the aciquisition from each station of a tunnel section (from pier to pier, excluding the pavement) made of slightly overlapping images (side lap).

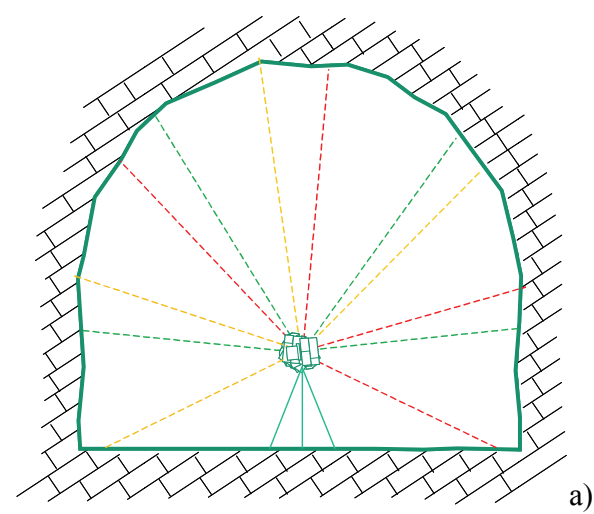

a)

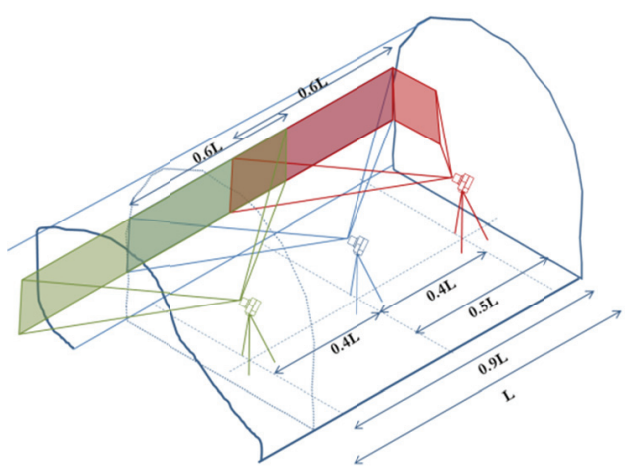

b)

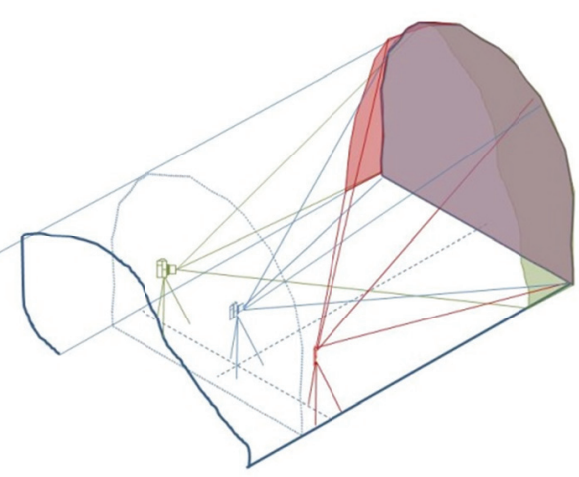

c)

Figure 1. Image sequence acquisition along the tunnel and at the excavation front

Once the transversal strip is shot, the tripods moves on by a longitudinal base length and acquires a new transversal strip (figure 1.b). The new one overlaps with the previous by the predefined percentage. A longitudinal strip is therefore made by 
all images acquired from the same stop of the rotation device from the different stations along the tunnel axis. Once the recently excavated section of the tunnel is surveyed, the excavation front is also surveyed by a minimum of three images (figure 1.c).

Once a new section is cleared after the blast, reference marks are painted on the piers and surveyed to check the progress of the drilling machine according to plan. They can easily be recognized in the image sequence and therefore used as control points for the orientation of the block of strips. This allows the progressive georeferencing of blocks along the tunnel, consistent with the tunnel reference system, without additional survey work that may interfere with the excavation.

\subsection{Image block design and simulation}

In a preliminary stage a simulation has been carried out to test the feasibility of the method. A tunnel section $10 \mathrm{~m}$ long, $8 \mathrm{~m}$ wide and $7.2 \mathrm{~m}$ high with rough surfaces has been generated in a virtual environment and draped with a photorealistic texture. This is roughly the amount of advance after each blast in the mining tunnel under study. Finally, artificial illumination reproducing the one available on site has been introduced. The synthetic block is made of three transversal strips with longitudinal baselenght of $2.1 \mathrm{~m}$; the survey of piers and vault is completed by 6 images spaced by $36^{\circ}$. Overall, therefore, the block is made of 6 longitudinal strips of 2 models each, plus 3 images of the front.

Synthetic images were generated and processed according to the pipeline described in the next section; an artificial DSM has been generated by image correlation. The results, though clearly obtained under ideal conditions, allowed to draw useful hints for the realization of the panoramic head and the strong influence of rock texture and illumination on the outcome.

\section{IMAGE PROCESSING DESCRIPTION}

\subsection{Image block orientation}

Many approaches have been developed in the past years in the photogrammetric and $\mathrm{CV}$ communities for the automated orientation of large and complex image sequences. The former discipline focuses primarily on precision and reliability of the results (Pierrot-Deseilligny et al., 2011). The latter is more concentrated in the orientation of large blocks (thousands of images) taken with un-calibrated cameras without any metric purpose except 3D visualization and image browsing (Snavely et al., 2008).

The main problems preventing the presence of reliable and precise commercial approaches are the presence of convergent images, unpredictable baselines and scale variations, lighting changes, repetitive patterns, homogeneous textured areas, etc. In this case, however, the geometric configuration of the block is quite regular: the base-length between consecutive images are always approximately the same and can be contained to grant a good overlap along the sequence, helping the identification of good tie points; all the images have a normal pose with respect to the object, basically analogous of that in an aerial block; the object usually presents, at this image scale, very good and contrasted elements that makes the tie point reconnaissance easy and very fast.

Consequently the automatic orientation approach has been designed based on simple SfM algorithms already developed by our research group for other, more demanding, applications: the orientation strategy follows a simple Feature Based Matching (FBM) procedure at the end of which the operator has only to identify the Ground Control Points (GCP) needed to georeference the image block.

Most FBM techniques used for image orientation are based on the use of detector/descriptor operators. The detector is capable of finding interest points in the images, while the descriptor associates a vector of information to each single detected point. As shown in (Roncella et al., 2011) the use of a scale-invariant feature extractor/descriptor, in this particular framework, is essential to obtain good results: as far as straight tunnel section are considered the use of any interest operator/descriptor is unimportant; due to the high overlap between consecutive images, and thanks to the high regularity in the block geometry and very good image texture, any strategy reach very good results. Nonetheless, sometimes, on the side walls of the tunnel apertures and intersection with other galleries are present: in that case the perspective changes between consecutive images are much higher and a non-scale invariant operator can lead to unpredictable results.

For this reason a SURF operator/descriptor has been implemented in the FBM routine: the SURF operator is partly derived from the SIFT detector and uses an integer approximation to the determinant of the Hessian, which can be computed very fast with integral images: while the SIFT operator is computationally very heavy, the SURF operator has a computational load comparable to that of the Harris operator (commonly used in many SfM strategies, even if it's only rotation invariant). Moreover, its scale invariance properties should grant higher repeatability compared to Harris.

To select the corresponding features by comparing directly the values of the descriptors, an exhaustive search along the lists can be performed; the computational load can be very high, though, due to the large number of features usually extracted by the operator. It is more efficient to use approximate methods like the kd-tree (Beis et al., 1997), that are faster but still deliver extremely reliable results.

As far as the epipolar geometry computation is concerned, rather than estimate the fundamental matrix, the 5-point calibrated relative orientation algorithm proposed by Nister (2004) has been implemented. Compared to the fundamental matrix, it offers a more straightforward approach that exploits the fact that, in practice, in all photogrammetric surveys the interior orientation elements (as well as image distortion parameters) are known.

The interior orientation is implicitly forced into the estimation using normalized image coordinates and obtaining directly the essential matrix. The mathematical model is more complex (it uses a $10^{\text {th }}$ degree polynomial instead of the $3^{\text {rd }}$ degree one for the fundamental matrix estimation); nonetheless, since it uses a more correct deterministic model, the estimation with the RANSAC algorithm achieves a higher inlier percentage: being the iterations dependent on the inlier percentage, the method is computationally more efficient than the old one.

We decided also to enforce the interior orientation during the three view geometry estimation. In this case it's much harder to implement the interior orientation parameters inside the trifocal tensor estimation: instead of using the trifocal tensor (Hartley et al., 2000) a RANSAC bundle block adjustment using just three images is used: six points are extracted randomly from the correspondences filtered out in the previous stages and the relative orientations parameters between a reference image and each of the two others images are computed; using the 6 points the three images are referred to the same object space; then, fixing the orientation parameters, all the other point correspondences are triangulated and image projection residuals are computed: all the points with a mean projection residual lower than a specified threshold are considered inlier. Maybe it can be less time consuming a strategy where the orientation 
parameters computed from the essential matrix stage are directly used: in this case the outlier can be filtered during the object space referencing of the triplets. Anyway the trifocal stage is usually very fast since the outlier percentage tends to be dramatically low: just few iterations are required to asses a good orientation solution.

At the same time the algorithm already provides a metric reconstruction of the triplets that can be easily concatenated afterwards.

At the end of the image sequence orientation stage all sequences acquired along the tunnel must be tied together. In this case the link between adjacent strips are made extremely easy thanks to the particular geometry of the images acquired from the same photo-station: approximately the acquisition device, at each photo-station location, rotates the camera around an horizontal axes that passes through the perspective center of the camera itself; the transformation from the image spaces of all the frames acquired in that position is well-approximated by an homography that depends on the rotation angle between the images:

$$
H=K R K^{-1}
$$

Where $\mathrm{H}$ is the transformation matrix (using homogenous coordinates) representing the homography between two images, $\mathrm{K}$ is the calibration matrix of the camera, which contains all the interior orientation parameters, and $\mathrm{R}$ is the relative rotation matrix between the two images.

Since the rotation angle between adjacent images is enforced by the acquisition device, once the interior orientation parameters are known, the homography approximating the transformation between them can be easily computed. The SfM strategy, then, tries to link the tie points on one image with the other in the other image: that should lead to the highest level of tightening between the strips since the tie points extracted so far are tracked for at least 3 images along the image sequences. If the points extracted are not enough however, using an Area Based approach, the homologous points corresponding to the tie points on one sequence are detected by an image correlation algorithm (LSM, Gruen, 1985).

At the end of the procedure a final bundle block adjustment with all the images (and with the GCP identified by the operator) is performed.

\subsection{DSM generation}

The DTM generation program Dense Matcher being developed at the University of Parma since 2006, implements both the Least Squares Matching (LSM) method (Gruen, 1985) and the Multiphoto Geometrically Constrained Matching (MGCM) method (Gruen et Baltsavias, 1988). The former is a wellknown local area-based method, where a linear radiometric transformation and an affine mapping are introduced to minimize the squared sum of the grey values differences between the patch image and the template image. The latter further extend the method coupling LSM with the geometric constraint of the collinearity equation; the method allows to accommodate any number of images available, exploiting image redundancy and providing greater reliability of the solution. As input data the program requires interior and exterior orientation parameters of the images and seed points that approximate the object surface to densify.

In most cases the seed points may be taken from the output of the image block orientation procedure of Section 3.1, that are normally dense and well distributed over the image format. Should it be necessary, a preliminary feature based extraction and matching is performed by using again the SURF operator and the epipolar constraint to filter out possible mismatches. The matching procedure is embedded in an image pyramid workflow and implements a parallel dense matching procedure. Images may undergo to epipolar resampling to improve efficiency and computational speed; a number of parameters (such as template window size, image correlation method, acceptance threshold, output point spacing, etc.) can be set to control the processing. Dense Matcher has been used and tested extensively in DTM generation of rock faces as well as in close range on cultural heritage artworks with multi-photo coverage.

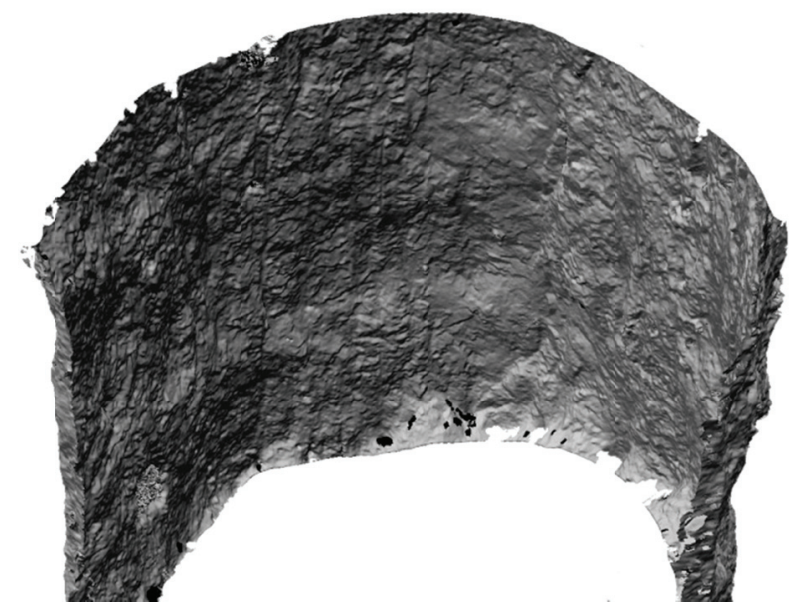

Figure 2. DSM of a tunnel section.

\subsection{Orthophoto production}

At the end of the DSM generation the data collected should be simplified to be analyzed and queried more easily by the geologists. To this aim the imagesi used during DSM generation are reprojected to generate an orthophoto of the inner surface of the tunnel. Since the geometry of the tunnel is generally complex, a simplified model of the gallery is required to allow the mapping of its intrados; in particular the geometry is composed of two planar surfaces approximating the abutments which support a semi-circular vault (see figure 3): every DSM point under the upper part of the abutments are projected orthogonally on the planar part of the simplified geometry, while the DSM portion whose height is higher than the abutments are projected along the radius direction of the circular vault.

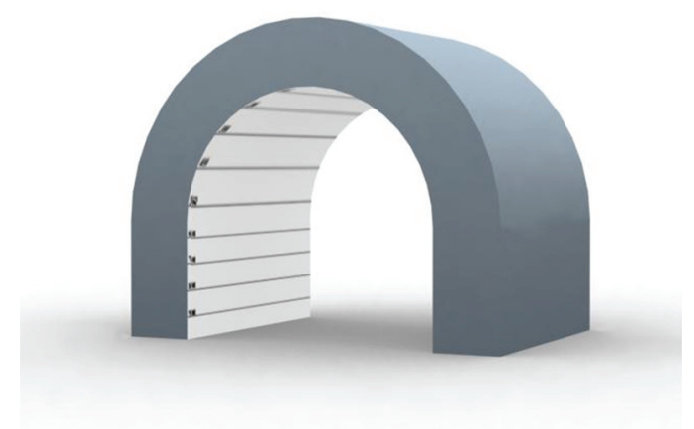

Figure 3. Simplified tunnel mapping geometry

A software to produce the orthophoto of the intrados of the gallery was proprietary developed; its workflow is as follow: first all the DSM points are mapped on the simplified tunnel geometry: the dimension of the simplified model are derived 
from the mean tunnel design dimensions (height of the abutments, maximum height of the vault, width of the gallery, etc.) and it's oriented so that its axis of symmetry coincide with those of the DSM; for every projected point the length of the projection is calculated (i.e. the distance from the simplified planes representing the abutments or the distance from the circular surface representing the gallery vault); a regular grid of points, representing the pixels in the final orthophoto, is generated onto the development of the inner surface of the simplified model; for every grid point the aforementioned length of projection is interpolated from the DSM data using a Delauney triangulation; inverting the projection process for every pixel the corresponding DSM interpolated position is computed; finally the interpolated points are projected on the sequence images allowing the determination of the color to be assigned to each orthophoto pixel.

In figure 4 an orthophoto representation of a $40 \mathrm{~m}$ tunnel section is presented.

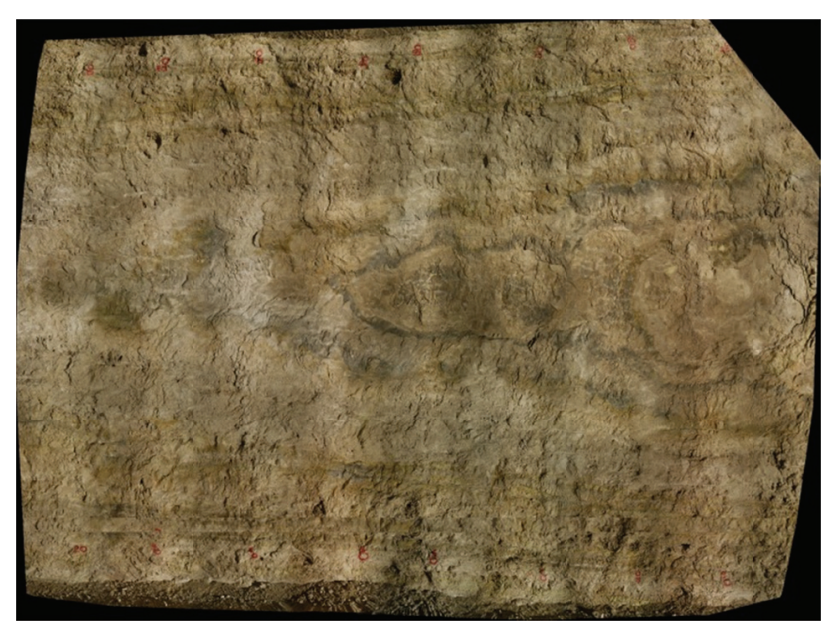

Figure 4. Orthophoto of a $40 \mathrm{~m}$ tunnel section

\section{THE GEOSTRUCTURAL SURVEY}

\subsection{Rockscan}

The geostructural survey of the discontinuities is performed with the Rockscan program, developed at the University of Parma, which allows the extraction of the orientation and the position of discontinuity surfaces as well as the measurement of discontinuity traces, on the basis of data obtained from a photogrammetric survey (Ferrero et al., 2009).

The program can operate either in fully automatic or in semiautomatic (interactive) mode. The former mode (Roncella and Forlani, 2005) is not suited for the analysis of tunnels or artificial benches, because the identification of discontinuity planes is purely based on a segmentation of the point cloud in plane regions; as pointed out above, the rock surface in the tunnel is disturbed by the excavation operations, so only some plane surfaces actually belong to real discontinuities. In other words, the identification of the planes must be supervised by an operator.

In interactive mode, through a GUI, the operator selects an area on the orthophoto, that are registered with respect to the point cloud. By drawing a polyline enclosing one or more discontinuities, a subset of the point cloud is selected and passed to the plane segmentation routine. The RANSAC algorithm is used iteratively to further segment this subset in disjoint sets, each corresponding to a discontinuity plane.
A first iteration of the algorithm selects the plane with the maximum number of inliers, i.e.the points at a distance to the plane less than the specified acceptance threshold.

Discarding the inliers from the point cloud, a new RANSAC step is executed and a new plane extracted; and so on. Ideally, the procedure would progressively select all planes, from the largest (in terms of number of inliers) to the smallest, until all points have been assigned or no more planes with a significant number of inliers can be found.

The user can specify the appropriate threshold for Ransac computation, depending on level of discretization of the DSM: while with rock faces values in the range from 10 to $20 \mathrm{~cm}$ are used in most cases, in tunnels the range is from 1 to $3 \mathrm{~cm}$.

The attitude data of the planes are expressed through the dip and the dip direction of the maximum dip line of the plane, derived from the normal to the plane. Each extracted planar surface is classified in terms of goodness of fit (mean square error), cardinality, location of the gravity centre and finally dip and dip direction.

Any image of the block depicting a given rock section can be used for segmentation; this allows the operator to look from different directions to the rock and therefore to find out the emerging planes of different fannilies, which may be clearly seen in some frames while being completely hidden in others. A large number of planes can be measured in a short period of time, which allows the structural layout of the entire excavation wall to be studied (including the vault), thus obtaining a statistically representative data sample.

\subsection{Results}

In the case study presented here the photogrammetric survey covered a stretch of tunnel of ca. $130 \mathrm{~m}$. The gallery crosssection has abutments about $3 \mathrm{~m}$ high and a semicircular vault with a radius of about $8 \mathrm{~m}$. The resolution required for the DSM was $5 \mathrm{~mm}$, while the accuracy needed to achieve enough information on the small rock discontinuities was of a few millimeters in each model. A Nikon D100 camera (6 Mpx) with a focal length of $18 \mathrm{~mm}$ was used for the survey providing an object resolution of $2 \mathrm{~mm} /$ pix on the abutment and $3 \mathrm{~mm} /$ pix on the vault.

Using the device and the imaging technique shown in Figure 1, so that the appropriate overlap between the longitudinal strips can be obtained, a section of the tunnel has been surveyed. For each tripod station, 6 images were taken, each covering approximately a $12 \times 8 \mathrm{~m}$ patch of the object. At the end of the procedure 6 longitudinal strips (with $80 \%$ longitudinal overlap and about $20 \%$ side-lap) were produced. A total of ca. 110 images per strip (i.e. 660 images in total) were captured (see figure 5 where just the first 40x6 images were shown).

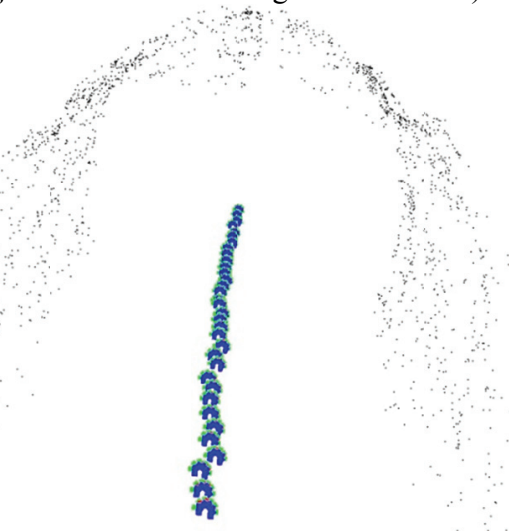

Figure 5. The first 40 photo-station oriented in the case study. 
As mentioned in Section 3.1, exploiting the approximate homography between strips to connect them in a single block, the points extracted in the overlap area of two longitudinal strips are transferred to the adjacent one to tie the strips together.

After the registration of all the images with a bundle adjustment and the successive DSM generation, a detailed orthophoto of the internal surface of the tunnel was created; the orthophoto relative to a section of about $40 \mathrm{~m}$ is shown in figure 4 .

\section{CONCLUSIONS}

The technical feasibility of performing geostructural surveys where discontinuity planes and traces are measured on high resolution DSM and oriented images has been shown for some time. The challenge is today to find technical solutions that allows the method to be applied advantageously in as many cases as possible. The results illustrated in this paper show that an effective operational pipeline can be set that allows minimal disruption of the mining operations, though guaranteeing correct results from the geostructural survey. In addition, consistent georeferencing and documentation with oriented images foresees the progressively builds up of a valuable database.

Further progress in data acquisition speed and safety of operation might be achieved by installing the camera and the device on the drilling machine, though this require a specific remote controlling by actuators of the rotating device and of the camera.

\section{REFERENCES}

3DM Analyst Mapping Suite. Product of Adam Technology, Perth, Australia

http://www.adamtech.com.au/3dm/Analyst.html

Bay, H., Ess, A., Tuytelaars, T., Van Gool, L., 2008. SURF: Speeded Up Robust Features. Computer Vision and Image Understanding (CVIU), Vol. 110, No. 3, pp. 346-359.

Beis, J.S., Lowe, D.G., 1997. Shape indexing using approximate nearest-neighbour search in high-dimensional spaces. In: Proc.of CVPR 1997, pp. 1000-1006.

Crosta, G. (1997): Evaluating rock mass geometry from photographic images. Rock Mech.Rock Engng., 30 (1), pp. 3558.

Fekete, S., Diederichs, M., Lato, M., 2009. Geotechnical applications of lidar scanning in tunneling. ROCKENG09: Proc. of the 3rd CANUS Rock Mechanics Symposium, Toronto May 9-15. ed. M. Diederichs and G. Grasselli. Paper 3987

Ferrero A.M., Forlani G., Roncella R., Voyat H. I. (2009). Advanced Geostructural Survey Methods Applied to Rock Mass Characterization. Rock Mech Rock Eng., 42: pp. 631-665.

Grün, A., 1985. Adaptive least squares correlations: a powerful matching techniques. South African J. Photogramm. Remote Sensing and Cartography, 14(3), pp175-187.

Grün, A., Baltsavias, E.P., 1988. Geometrically constrained multiphoto matching. PE\&RS, 54(5), pp. 633-641.

Hadjigeorgiou, J., Lemy, F. (2005): Développement d'un système d'analyse d'images pour la caractérisation des massifs rocheux. Rapport soumis à l'Institut de recherche Robert-Sauvé en santé et sécurité du travail (IRSST). (Rapport R-414) : 46 pp.
Hartley, R., Zisserman, A., 2000. Multiple View Geometry in Computer Vision. Cambridge University Press, Cambridge, pp. $1-496$.

Nakai, T. et al., 2003. Support design of river crossing tunnel using keyblock concept and its validation by monitoring of the countermeasure by digital photogrammetry at Suzuka tunnel. Development and Application of Discontinuous Modelling for Rock Enginnering, Proceedings of the 6th International Conference on Analysis of Discontinuous Deformation, 5-8 October 2003, Trondheim, Norway. Ed Taylor \& Francis, 2003.

Nister, D., 2004. An efficient solution to the five-point relative pose problem. IEEE T, Pattern Anal., 26(6): 756-770.

Nuttens,T. et al., 2010. High Resolution Terrestrial Laser Scanning for Tunnel Deformation Measurements. FIG Congress 2010 Facing the Challenges - Building the Capacity. Sydney, Australia, 11-16 April 2010

Pierrot-Deseilligny, M., Clery, I. APERO, an open source bundle adjustment software for automatic calibration and orientation of set of images. Int. Archives of Photogrammetry Remote Sensing and Spatial Information Sciences, 38-5/W16, on CD-ROM (2011).

Roncella, R., Re, C., Forlani, G. Comparison of two structure and motion strategies. Int. Archives of Photogrammetry, Remote Sensing and Spatial Information Sciences, 2011, 38(5/W16), on CD-ROM. ISPRS Int. Workshop 3D-ARCH 2011, Trento, Italy.

Roncella R., Forlani G. (2005). Extraction of planar patches from point clouds to retrieve dip and dip direction of rock discontinuities. Workshop 'Laser scanning 2005', Enschede (NL) 12-14 Sept. 2005. The International Archives of the Photogrammetry, Remote Sensing and Spatial Information Sciences, vol. XXXVI part 3/W19, pp. 162-167.

Slob S., Hack H.R.G.K., van Knapen B., Turner K. and Kemeny, J. (2005) A method for automated discontinuity analysis of rock slopes with 3D laser scanning. In: Transportation Research Record, 1913(2005)1, pp. 187-208.

Snavely, N., Seitz, S.M., Szeliski, R., Modelling the world from internet photo collections. International Journal of Computer Vision, 80(2), 189-210 (2008)

Somervuori, P., Lamberg, M., 2009. "Modern 3D photogrammetry method for rock mechanics, geological mapping, 3D model and documentation of open pit faces and tunnels." http://www.wspgroup.com/en/Welcome-to-WSPFinland/Services/Services-Container/mine-Planning/

Tsoutrelis, C.E., Exadactylos, G.E., Kapenis, A.P. (1990): Study of the rock mass discontinuity system using photoanalysis. Mechanics of Jointed and Faulted Rock, Rossmanith (éd.), Balkema, Rotterdam, pp. 103-112. 